\title{
Ion Irradiation Damage in Titanate Ceramics as a Function of Dose
}

\author{
M.G. Blackford*, G.R. Lumpkin", K.L. Smith, H. Li ${ }^{*}$ and M. Colella* \\ *Materials Division, Australian Nuclear Science and Technology Organisation, PMB 1, Menai, NSW \\ 2234, AUSTRALIA
}

The immobilisation of high level radioactive waste (HLW) in a solid waste form with subsequent geological isolation from the biosphere is one of the preferred strategies for the management of HLW. The radiation resistance of a waste form may affect the long-term aqueous durability of the waste form. Therefore, it is crucial to understand the radiation damage process and its consequences (amorphisation, cation disordering, volume expansion, cracking, etc.).

Alpha decay is the dominant radiation damage mechanism in actinide-bearing HLW waste forms. The amount of damage accumulates over time, resulting in regions of amorphous material and eventually to complete amorphisation at a critical dose, $D_{c}$. This process can be simulated using energetic heavy ion irradiation. In this study, we report on radiation damage effects in a pyrochlore $\left(\mathrm{Gd}_{2} \mathrm{Ti}_{2} \mathrm{O}_{7}\right)$ and a Nd-doped zirconolite $\left(\mathrm{Ca}_{0.8} \mathrm{Nd}_{0.2} \mathrm{Zr}_{1} \mathrm{Ti}_{1.8} \mathrm{Al}_{0.2} \mathrm{O}_{7}\right)$. Both of these phases are important hosts for actinides in ceramic waste forms.

The critical doses of $1 \mathrm{MeV} \mathrm{Kr}^{2+}$ ions for amorphisation of the pyrochlore and zirconolite at room temperature were determined by irradiating specimens using the IVEM-Tandem Facility at Argonne National Laboratory, USA. Progression of damage was followed in situ by alternately irradiating and examining specimens using TEM and selected area diffraction. In this study, we determined critical amorphisation doses of $2.5 \times 10^{14}$ ions $/ \mathrm{cm}^{2}$ for pyrochlore and $3.9 \times 10^{14}$ ions $/ \mathrm{cm}^{2}$ for zirconolite.

Additional samples were irradiated to fractions of $D_{c}(0.1,0.2,0.3, \ldots, 0.9)$. These were examined using high-resolution electron microscopy, selected area electron diffraction and electron energy loss spectroscopy (EELS) to monitor changes in the structure and bonding in pyrochlore and zirconolite as a function of ion dose. Preliminary results are presented below for the pyrochlore.

Figure 1 shows two series of selected area electron diffraction patterns (SADPs) recorded as a function of dose from the [0 (bar)11] zone axis (1a to 1e) and with the sample tilted approximately $9^{\circ}$ off the zone axis where the (111) systematic row of reflections is prominent (1f to $1 \mathrm{j}$ ). It is apparent that the diffracted amplitude of the diffuse rings is greater in the SADPs taken in the systematic row orientation up to about 0.6-1.0 Dc. This is due to strong dynamical scattering effects in the TEM and enables one to detect the presence of amorphous domains at a lower fractional dose when compared with the zone axis SADPs.

Previous authors have postulated that changes in the intensity of the superlattice reflections relative to the sublattice reflections indicate that pyrochlore undergoes a structural change to the fluorite structure (via cation mixing) before becoming amorphous. In this study, SADP simulations indicate that as contiguous volumes of crystalline material become smaller with increasing dose, the amplitudes of the superlattice reflections decrease rapidly relative to the sublattice. Therefore, the SADPs alone do not provide evidence for Gd-Ti cation disordering over the A and B sites (also crystal chemically unlikely). 
Figure 2 illustrates the change in the EELS spectrum of the $\mathrm{Ti}_{2,3}$ and $\mathrm{O} \mathrm{K}$ edges from the pyrochlore after a critical dose of $1 \mathrm{MeV} \mathrm{Kr}^{2+}$ ions. Amorphisation results in a slight energy shift and reduced splitting of the edges. These results are consistent with previous EELS work on unirradiated and irradiated zirconolite, suggesting that there is a reduction in the coordination number of Ti. In fact, the EELS spectrum of Ti in the pyrochlore irradiated to $1.0 \mathrm{Dc}$ is remarkably similar to that of $\mathrm{Ti}$ in the mineral fresnoite. The measured energy shift of $1.0 \mathrm{eV}$ indicates that the $\mathrm{Ti}$ coordination is in part reduced from 6 to 5 in the fully amorphous sample (reduced coordination is inconsistent with cation disordering at an intermediate dose). Note that at this stage, we cannot rule out a small component of $\mathrm{Ti}^{3+}$ in the spectra.

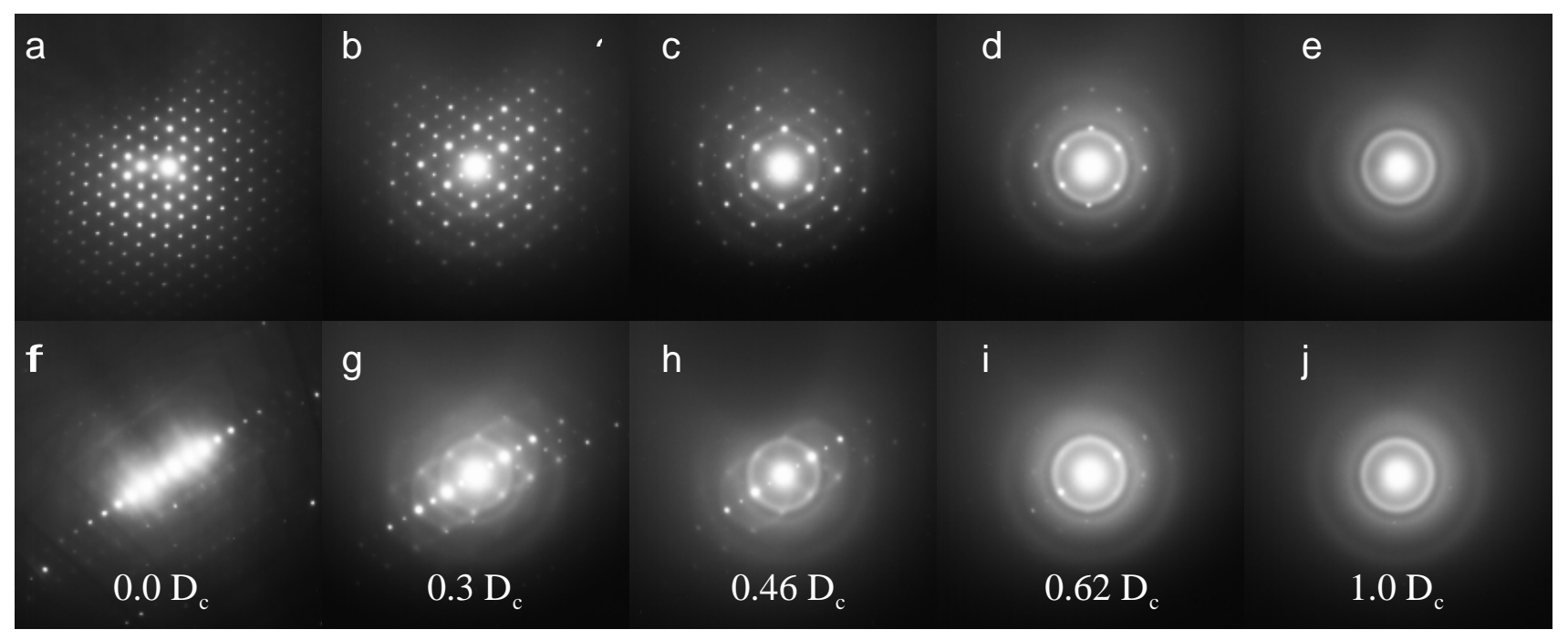

Fig. 1 Selected area electron diffraction patterns from the pyrochlore [0(bar)11] zone axis (a to e) and the (111) systematic row ( $f$ to $j$ ) as a function of ion irradiation dose (in fractions of $D_{c}$ ).
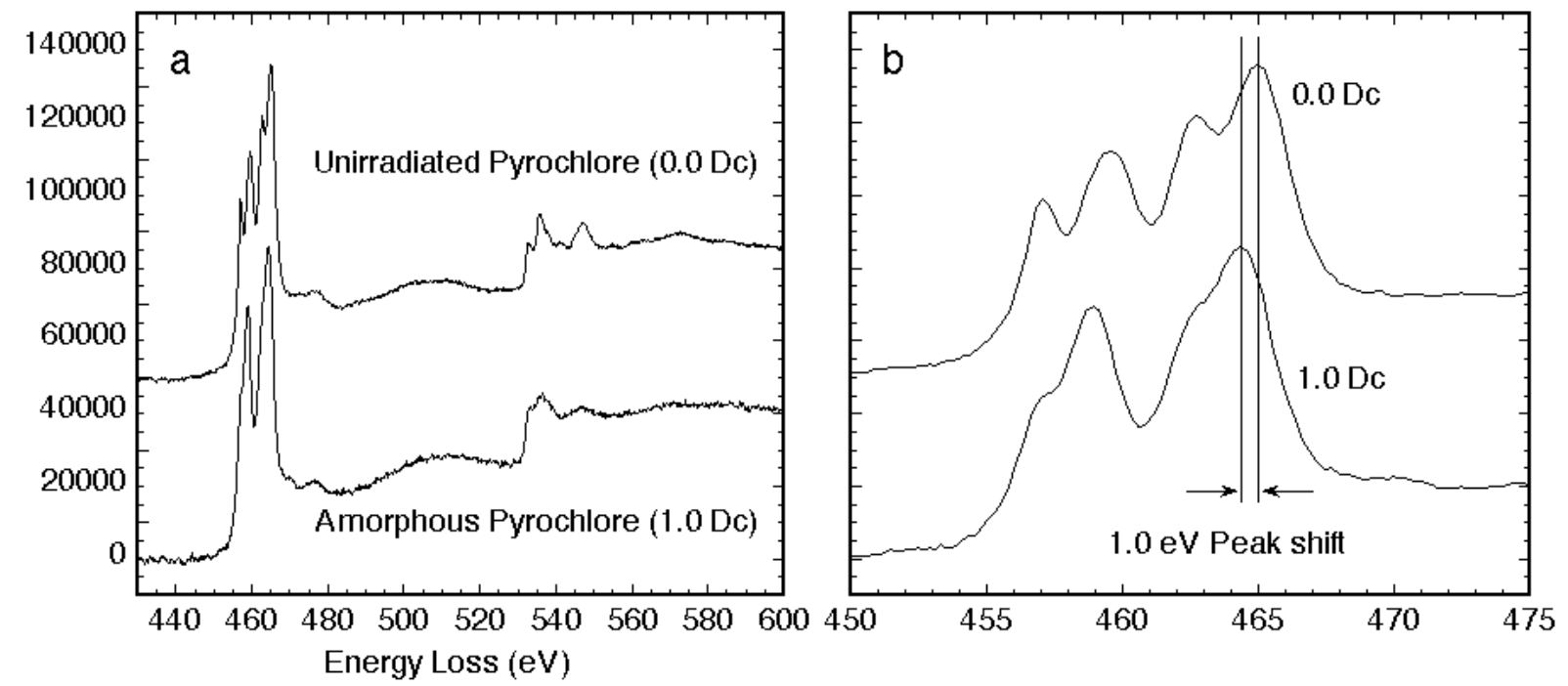

Fig. 2. a) EELS spectra from pre-irradiated and full amorphous pyrochlore showing significant alteration of the $\mathrm{Ti}_{2,3}$ and $\mathrm{O} \mathrm{K}$ edges due to ion irradiation. b) Expanded plot of the $\mathrm{Ti}_{2,3}$ edges showing reduced peak splitting as well as a small but significant peak shift. 